\title{
A SERIES REPRESENTATION FOR RIEMANN'S ZETA FUNCTION AND SOME INTERESTING IDENTITIES THAT FOLLOW
}

\author{
MichaEL MiLgRAM
}

Abstract. Using Cauchy's Integral Theorem as a basis, what may be a new series representation for Dirichlet's function $\eta(s)$, and hence Riemann's function $\zeta(s)$, is obtained in terms of the Exponential Integral function $E_{S}(i \kappa)$ of complex argument. From this basis, infinite sums are evaluated, unusual integrals are reduced to known functions and interesting identities are unearthed. The incomplete functions $\zeta^{ \pm}(s)$ and $\eta^{ \pm}(s)$ are defined and shown to be intimately related to some of these interesting integrals. An identity relating Euler, Bernouli and Harmonic numbers is developed. It is demonstrated that a known simple integral with complex endpoints can be utilized to evaluate a large number of different integrals, by choosing varying paths between the endpoints.

Mathematics subject classification (2010): 11M06, 11M26, 11M35, 11M99, 26A09, 30B40, 30E20, 33С20, 33B20, 33B99.

Keywords and phrases: Riemann zeta function, Dirichlet eta function, alternating zeta function, incomplete zeta function, generalized exponential integral, Bernoulli numbers, Euler numbers, harmonic numbers, infinite series, evaluation of integrals.

\section{REFERENCES}

[1] Michael Milgram, An integral equation for Riemann's Zeta function and its approximate solution, Abstract and Applied Analysis, /2020/1832982(1832982), May 2020,

https://doi.org/10.1155/2020/1832982.

[2] AndRe LeClair, An electrostatic depiction of the validity of the Riemann Hypothesis and a formula for the N-th zero at large N, Int. J. Mod. Phys., A28:1350151, 2013, also available from http://arxiv.org/abs/1305.2613v3.

[3] M. L. GLASSER, A note on the Riemann $\xi$-function, 2019, available from https://arxiv.org/abs/1901.07011.

[4] M. S. Milgram, The Generalized Integro-Exponential Function, Mathematics of Computation, 1985, available from https://www . ams.org/mcom/1985-44-170/S0025.../S0025-5718-1985-0777276-4.pdf.

[5] R. B. PARIS, A generalization of an expansion for the Riemann Zeta function involving incomplete Gamma functions, Applied Mathematical Sciences, 3 (60): 2973-2984, 2009, also available from https://core.ac.uk/download/pdf/228177306.pdf.

[6] W. H. L. RusselL, On certain integrals, Proc. Royal Soc. London, 25 (176), 1876.

[7] Tewodros Amdeberhan And Victor Moll, A dozen integrals: Russell-style, 2008, available from https://arxiv.org/abs/0808.2692.

[8] MARK W. COFFEY, Generalizations of Russell-style integrals, June 2018, https://arxiv.org/abs/1806.07962.

[9] MARK W. COFFEY, Integrals in Gradshteyn and Ryzhik: Hyperbolic and Trigonometric functions, 2018, https://arxiv.org/abs/1803.00632.

[10] E. C. Titchmarsh and D. R Heath-Brown, The Theory of the Riemann Zeta-Function, Oxford Science Publications, Oxford, Second edition, 1986.

[11] Michael Milgram, Notes on a paper of Tyagi and Holm: A new integral representation for the Riemann Zeta function, 2007, available from https://arxiv.org/abs/0710.0037, also available from https://www.researchgate.net/publication/1767498. 
[12] ÖMER KÜÇÜKSAKALLI, A recurrence relation for Bernoulli numbers, Hacettepe Journal of Mathematics and Statistics, 42 (4): 319-329, 2013.

[13] F. W. J. Olver, D. W. Lozier, R. F. Boisvert, and C. W. Clark, editors, NIST Handbook of Mathematical Functions, Cambridge University Press, New York, NY, 2010, print companion to [26].

[14] Michael Milgram, Some Additions to a family of sums and integrals related to Hurwitz' Zeta functions, Euler polynomials and Euler Numbers, (submitted for publication), 2021.

[15] IRA M. GeSsel, On Miki's identity for Bernoulli numbers, Journal of Number Theory, 110 (1): 75 82, 2005, Arnold Ross Memorial Issue, https://doi.org/10.1016/j.jnt.2003.08.010.

[16] Wolfram Research, Harmonic Number, retrieved from https://functions.wolfram.com/GammaBetaErf/HarmonicNumber/07/01/01/0001/.

[17] V. H. Moll And C. VignAt, Integrals involving Bernoulli and Euler polynomials, SCIENTIA, Series A, Mathematical Sciences, 30: 55-78, 2020.

[18] I. S. Gradshteyn And I. M. Ryzhik, Tables of Integrals, Series and Products, corrected and enlarged Edition, Academic Press, 1980.

[19] Wolfram Research, Champagne, Illinois, Mathematica, version 12, 2019.

[20] Maplesoft, a division of Waterloo Maple Inc., version 2018, Maple.

[21] H. M. Srivastava And Junes ang Choi, Zeta and q-Zeta Functions and Associated Series and Integrals, Elsevier, 32 Jamestown Rd., London, NW1 7BY, first edition, 2012.

[22] K. S. Williams AND N.-Y. ZHANG, Special values of the Lerch Zeta function and the evaluation of certain intgrals, Proceedings of the American Mathematical Society, 119 (1), 1993.

[23] J. M. BorWein, P. B. BorWein, AND K. Dilcher, Pi, Euler numbers, and asymptotic expansions, The American Mathematical Monthly, 96 (8): 681-687, 1989, available from www.cecm.sfu.ca/personal/pborwein/PAPERS/P45.pdf.

[24] F. W. J. Olver, Asymptotics and Special Functions, Academic Press, 1974.

[25] JINHEE YI, Theta-function identities and the explicit formulas for theta-function and their application, J. Math. Analysis and Applications, 292 (2): 381-400, 2004, https://doi.org/10.1016/j.jmaa.2003.12.009.

[26] NIST Digital Library of Mathematical Functions, http://dlmf .nist.gov/, Release 1.0.9 of 201408-29.

[27] Dan Romik, The Taylor coefficients of the Jacobi theta constant $\theta_{3}$, Ramanujan Journal, 52 (2): 275 290, 2020, https://doi.org/10.1007/s11139-018-0109-5, also available from arXiv e-prints, July 2018, arXiv: 1807.06130.

[28] M. S. Milgram, Integral and series representations of Riemann's Zeta function, Dirichlet's Eta function and a medley of related results, Journal of Mathematics, Article ID 181724, 2013, http://dx.doi.org/10.1155/2013/181724.

[29] A. Magnus, W. Oberhettinger, F. Erdelyi And F. G. Tricomi, Higher Transcendental Functions, volume 1, McGraw-Hill, 1953.

[30] Eldon R. Hansen, A Table of Series and Products, Prentice-Hall Inc., Englewood Cliffs, N. J., 1975.

[31] E. W. Weisstein, Dirichlet Beta Function, Mathworld - a Wolfram Web Resource., Dec. 2016, retrieved from http://mathworld.wolfram.com/DirichletBetaFunction.html.

[32] E. W. Weisstein, Log Gamma function, Mathworld - a Wolfram Web Resource., 2005, retrieved from https://mathworld. wolf ram. com/LerchTranscendent.html.

[33] Y. LUKE, The special functions and their approximations, Volume I, volume 53 of Mathematics in Science and Engineering, Academic Press, 1969.

[34] José LuIs LóPeZ AND Nico M. Temme, Large degree asymptotics of generalized Bernoulli and Euler polynomials, Journal of Mathematical Analysis and Applications, 363 (1): 197-208, 2010, https://doi.org/10.1016/j.jmaa.2009.08.034. 